Research Article

\title{
Computed Tomography Image under Optimized Iterative Reconstruction Algorithm to Analyze the Characteristics of Blood Flow Field in Cerebral Aneurysm before and after Stent Implantation
}

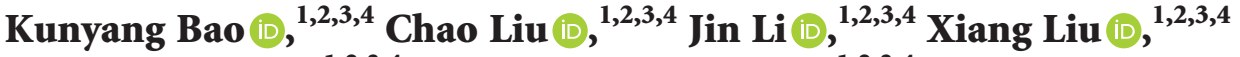 \\ Wenzhang Luo $\mathbb{D},,^{1,2,3,4}$ and Changren Huang $\mathbb{D}^{1,2,3,4}$ \\ ${ }^{1}$ Department of Neurosurgery, The Affiliated Hospital of Southwest Medical University, Luzhou 646000, Sichuan, China \\ ${ }^{2}$ Academician (Expert) Workstation of Sichuan Province, Luzhou 646000, Sichuan, China \\ ${ }^{3}$ Laboratory of Neurological Diseases and Brain Function, Luzhou 646000, Sichuan, China \\ ${ }^{4}$ Sichuan Clinical Research Center for Neurosurgery, Luzhou 646000, Sichuan, China
}

Correspondence should be addressed to Changren Huang; huangchangren6f@163.com

Received 15 August 2021; Revised 29 September 2021; Accepted 30 September 2021; Published 2 December 2021

Academic Editor: Gustavo Ramirez

Copyright ( 2021 Kunyang Bao et al. This is an open access article distributed under the Creative Commons Attribution License, which permits unrestricted use, distribution, and reproduction in any medium, provided the original work is properly cited.

In order to analyze the change characteristics of blood flow field in cerebral aneurysms before and after stent implantation, this study first constructed an optimized iterative reconstruction algorithm to reconstruct CT images of patients with cerebral aneurysms and used it to solve the problem of image sharpness. In addition, backprojection image reconstruction algorithm and Fourier transform analytic method were introduced. According to the CT images of cerebral arteries of patients, the lesions were presented in a three-dimensional and visual way through the reconstructed three-dimensional images, thus achieving the effects of simulation and simulation. The results showed that the sensitivity, specificity, and accuracy of the optimized iterative reconstruction algorithm were $90.78 \%, 83.27 \%$, and $94.82 \%$, which were significantly higher than those of the backprojection image reconstruction algorithm and Fourier transform analysis method, and the difference was statistically significant $(P<0.05)$. Before operation, the blood flow velocity in the neck of aneurysm was $7.35 \times 10^{-2} \mathrm{~m} / \mathrm{s}$, the exit velocity was $1.51 \times 10^{-1} \mathrm{~m} / \mathrm{s}$, and the maximum velocity appeared in the upstream part of the exit. After passing through the aneurysm, the blood flow velocity began to decrease gradually, forming a vortex at the top of the tumor. After stent implantation, the neck and outlet velocities of cerebral aneurysm were $9.352 \times 10^{-2} \mathrm{~m} / \mathrm{s}$ and $1.897 \times 10^{-2} \mathrm{~m} / \mathrm{s}$, respectively. The velocity of blood flow decreased after entering the aneurysm, and there was no vortex at the top of the aneurysm. Among the outlet velocities of arterial blood vessels, the velocity before stent implantation was significantly lower than that after stent implantation, and the difference was statistically significant $(P<0.05)$. Compared with prestent, the shear force distribution on the wall of cerebral aneurysm showed a significant decrease, and the difference was statistically significant $(P<0.05)$. To sum up, pelvic floor ultrasound based on hybrid iterative reconstruction algorithm has high accuracy in diagnosing the changes of blood flow field in cerebral aneurysms. The application of CT images in the diagnosis of cerebral aneurysms can objectively provide imaging data for clinical practice and has high application value.

\section{Introduction}

The cardiovascular and cerebrovascular diseases show the highest mortality rate in the world today. According to research reports, more than half of the deaths in the United
States each year are directly or indirectly caused by cardiovascular and cerebrovascular diseases [1]. In recent years, changes in people's diet have led to an increase in the incidence of cardiovascular and cerebrovascular diseases. This is also one of the fastest growing causes of death among 
Chinese residents, so the incidence of this disease is becoming younger and younger. With the progress of society and the improvement of people's living standards, more and more people choose to have cerebral aneurysm surgery after illness [2]. However, the traditional operation is too complicated, the risk of death is high, and the operation cost is expensive, which causes huge economic pressure on people. In view of the high risk and high cost of traditional surgery, it is very important to find simple, safe, and low-cost treatments [3]. With the development of intracranial aneurysm interventional materials and surgical methods, intravascular interventional therapy has become one of the important treatment methods for intracranial aneurysms, and its effect is equivalent to that of craniotomy [4]. The stent can play a supporting role, not only conducive to the dense embolization of the coil, but also conducive to endothelial growth and repair, and can improve the blood flow of the tumor carrier artery. Intracranial vascular stent implantation can affect the hemodynamics of aneurysms, reduce the recurrence rate of aneurysms by promoting the formation of carotid artery intima, and achieve the anatomical cure [5].

Nowadays, there are many ways and equipment to diagnose diseases. According to the different imaging principles, they can be divided into ultrasound imaging technology, computed tomography (CT) technology, magnetic resonance imaging (MRI) technology, and so on. These detection methods can be used to extract image data of tissues, organs, or systems inside the patient. It can significantly expand the scope of patient examination and improve the detection rate and accuracy of lesions [6]. CT has accurate feedback for the diagnosis of diseases and has high sensitivity and accuracy for determining the location of lesions and the size and number of lesions. It also exerts a reliable prompting effect on the tissue infiltration around cancer in the brain, so it is very suitable for collection and study the images of cerebral artery tumor [7]. CT diagnosis is helpful for constructing an accurate and individualized model. It is easy to collect data, and the diagnosis cost is relatively cheap, so it is widely used in cerebral artery tumors. Iterative reconstruction of CT image is an alternative method of image reconstruction, which is based on the process that CT detector receives the original data that can represent X-ray intensity, solves the pixel values in the matrix of the image, and restores the anatomical structure of human body [8]. Hybrid iterative reconstruction algorithm is composed of two denoising parts: iterative maximum likelihood restoration based on noise distribution and fitting the local structure model of image data that can greatly reduce irrelevant noise [9]. Therefore, this research will use the hybrid iterative reconstruction algorithm to reconstruct and analyze multislice spiral CT images, so as to improve the quality of CT images and the effect of auxiliary diagnosis and treatment.

In summary, an optimized HIR algorithm was constructed and applied to the image analysis of patients with intracranial aneurysms in this study. Subsequently, reverse engineering was adopted to establish a physical model of cerebral aneurysm, design an individualized intravascular stent model, and analyze the two-way fluid-solid coupling after stent implantation to obtain the velocity vector, streamline, and wall shear stress, total deformation, and pressure distribution of the aneurysm model, aiming to provide a theoretical basis for clinical analysis of cerebral aneurysm rupture mechanism.

\section{Materials and Methods}

2.1. Selection of Research Samples. In this study, 50 patients with cerebral aneurysms diagnosed by pathology from January 20, 2019, to February 15, 2020, were selected as the research objects, and all the intracranial CT images of these patients were collected based on the optimized hybrid iterative reconstruction algorithm. A male patient under 55 years old with intracranial aneurysm of internal carotid artery was selected for the study. The study had been approved by the Ethics Committee of hospital. The patient and his family members had known about the study and signed an informed consent form.

Inclusion criteria were the following: (1) patients diagnosed as cerebral aneurysms by early pathological and imaging diagnosis; (2) their ages were between 45 and 65 years old; (3) the patient not receiving other drugs and antibiotics in recent years; (4) patients with normal coagulation function and platelets.

Exclusion criteria were the following: (1) patients with other system or organ diseases; (2) patients who had not received cooperative treatment due to personal or other factors; (3) patients with incomplete clinical data and medical history information.

\subsection{Three-Dimensional (3D) Reconstruction of Cerebral} Aneurysm. Images were collected using GEAW4.6 workstation volume reconstruction (VR) and Maximum Intensity Projection (MIP) methods with a 64-slice spiral CT machine from General Motors. The iohexol contrast agent was $400 \mathrm{mg} \mathrm{I} / \mathrm{mL}$, and the total dose of high-pressure bolus injection was about $90 \mathrm{~mL}$. The layer thickness was set to $7 \mathrm{~mm}$, the layer spacing was $4 \mathrm{~mm}$, the reconstruction layer thickness was $0.6 \mathrm{~mm}$, the pitch was about $1.5 \mathrm{~mm}$, and the resolution was $1400 \times 750$.

The Digital Imaging and Communications in Medicine (DICOM) data were utilized to import the Mimics window and adjust the image display to dynamic area growth. The dynamic threshold range was undertaken as the boundary to extract the lesion and create a new mask. A 3D model of the CT image was reconstructed, which was trimmed and smoothed. The reverse engineering software was used to mesh the surface mesh of the aneurysm flow field and the surface of the aneurysm wall, respectively, and the poor quality mesh was repaired and the surface was smoothed and output. The specific effects could be observed in Figure 1.

\subsection{Other Theories}

2.3.1. Stent Implantation and Cerebral Aneurysm Fluid-Solid Coupling Solution Setting. The spiral stent developed in this study could meet the mechanical properties, such as radial 


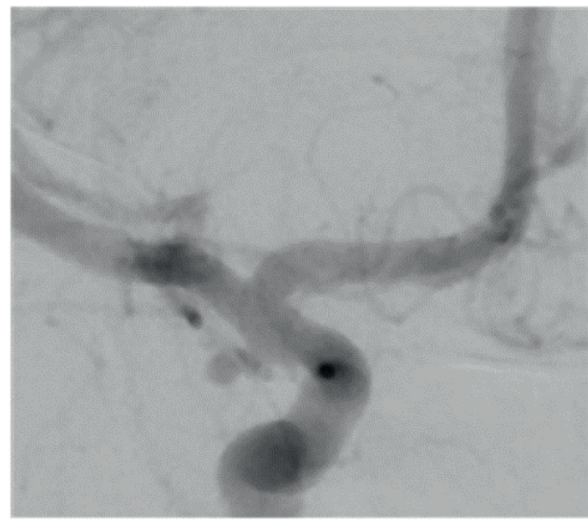

(a)

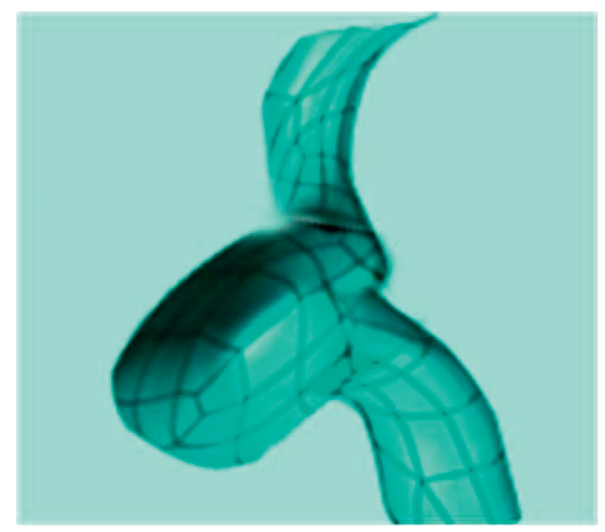

(b)

FIGURE 1: Internal carotid angiography showed right prechoroidal aneurysm (a); the cerebral aneurysm surface model optimized by reverse engineering $(b)$.

support force, surface coverage, expandability, and longitudinal shortening rate. The $3 \mathrm{D}$ modeling of the stent consists of four metal wires wound clockwise and four other wires wound together counterclockwise. According to literature reports, the diameter of the spiral circular cross section stent was generally $0.13 \mathrm{~mm}$, and the transmittance was about $78 \%$. Therefore, the circular cross section spiral stent was drawn based on the CT image data, and the personalized entity cerebral aneurysm model of the inner carotid artery was extracted, the blood was filled into the fixed model, and the blood and vascular stent were synthesized and cut to obtain the stent pressure blood model (as given in Figure 2).

\subsubsection{Cerebral Aneurysm Fluid-Solid Coupling Solution} Setting. In the fluid part, related study [10] showed that as long as the diameter of the tumor-bearing artery was greater than $0.5 \mathrm{~mm}$, Newtonian fluid can be used instead of the error caused by non-Newtonian fluid. In this study, the entrance diameter of the tumor-bearing cerebral artery was $2.5 \mathrm{~mm}$, the exit diameter was $1.8 \mathrm{~mm}$, and the exit diameter at the neck of the tumor was $1.25 \mathrm{~mm}$, which were all greater than $0.5 \mathrm{~mm}$. Therefore, a uniform, incompressible, viscous Newtonian fluid can be used instead of a non-Newtonian fluid to simplify the model. The blood density was set to $1000 \mathrm{~kg} / \mathrm{m}^{3}$, and the blood viscosity was set to $0.003 \mathrm{pa} / \mathrm{s}$. According to the Reynolds number Re $>\rho u r / \eta$ (1), $\rho$ represented the fluid density, $r$ represented the inner diameter of the blood vessel, and $\eta$ referred to the viscosity coefficient of the fluid. The calculated Reynolds number value satisfied $\operatorname{Re}<2000$. The turbulence may appear in the local area under some specific conditions, but the pinch blood flow of the cerebral artery was essentially the upper layer. The blood flow was simulated as adiabatic, incompressible, and unsteady stable Newtonian laminar flow in an elastic tube. The whole technology started from the static state with the initial value of the flow field at 0 and terminated when the stable value was satisfied [11].

In the setting of the properties and boundaries of the solid part, the geometric deformation of the arterial wall was a very complex nonlinear change process. In order to simplify the calculation, it can be assumed that the arterial wall and the tumor wall were linear, anisotropic, homoelastic materials, and the wall surface was set with no-slip conditions, which was to set the avoided speed to be 0 , the density to be $1100 \mathrm{~kg} / \mathrm{m}^{3}$, and the Young's modulus to be $2.8 \mathrm{MPa}$. The wall surfaces at both ends of the tube wall adopted fixed supports; the thickness and size of the cerebral artery wall were known, and the aneurysm wall was thinner than the artery wall, so the aneurysm wall thickness was selected as $0.1 \mathrm{~mm}$.

2.4. Construction of HIR Algorithm. HIR algorithm can denoise images well, improve image quality, and reduce radiation [12]. The working principle of the HIR algorithm was to reduce the noise in the front and rear projection domains. The principle is illustrated in Figure 3.

It was proved that the projector data received by the CT detector was very similar to the Gaussian distribution, and the mean and variance of the projection data after logarithmic transformation conformed to the following equation:

$$
R_{k}^{2}=A_{k} \times \exp \frac{B_{k}}{\eta}
$$

In the equation above, $R_{k}$ represented the mean value of the data received by the $k^{\text {th }}$ detector unit, and $R_{k}^{2}$ represented the variance of the data. In the traditional algorithm, the weighted least-squares lattice (WLSL) can be applied to reduce the image noise in the front and rear projection space:

$$
\begin{aligned}
& \phi(a)=(\hat{y}-a)^{\prime} \Sigma^{-1}(\hat{y}-a), \\
& \phi(v)=(\widehat{y}-l v)^{\prime} \Sigma^{-1}(\hat{y}-l v) .
\end{aligned}
$$

Equation (2) was the iterative equation of the front projection domain, and (3) was the iterative equation of the backprojection domain. $a=l v$ was the vector of the complete projection data set, which was estimated from the initial 


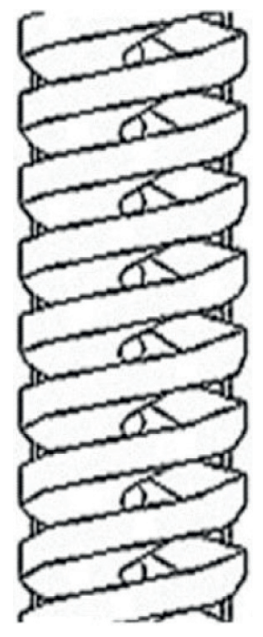

(a)

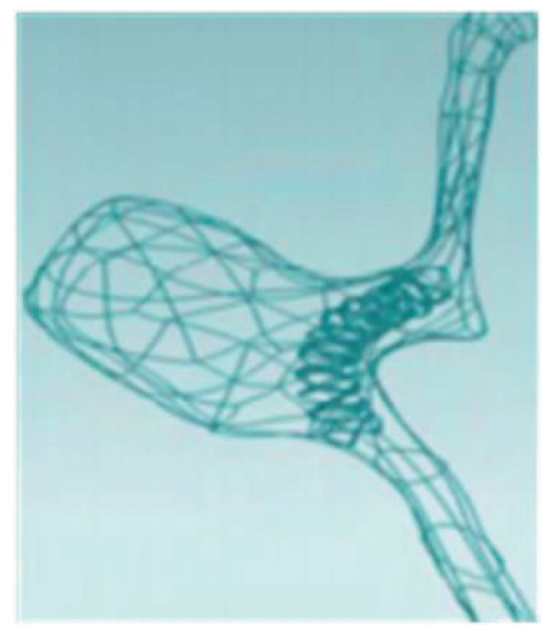

(b)

Figure 2: Spiral scaffold model (a); cerebral aneurysm model after stent implantation (b).

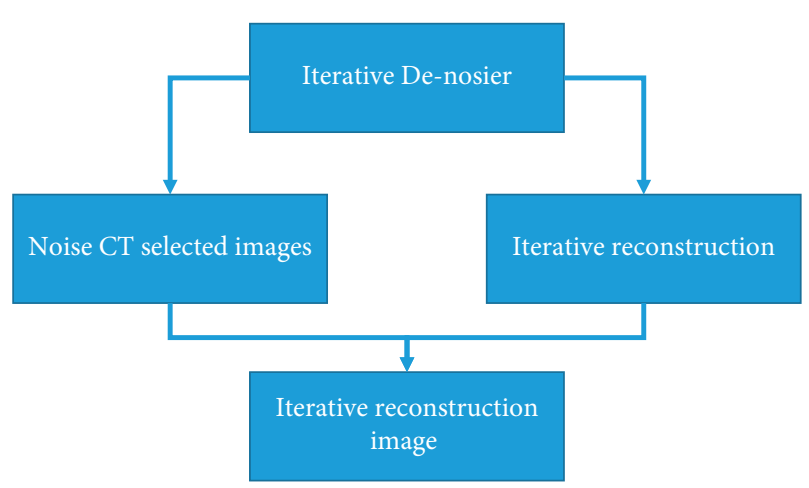

FIGURE 3: Schematic diagram for principle of HIR algorithm.

image, $v$ referred to the attenuation coefficient to be calculated finally, $l$ represented the projection matrix, and $y$ stood for the logarithmic result of the detector measurement data [13].

The penalty WLSL was introduced in the CT iterative algorithm because a slightly smooth penalty factor could improve the checkerboard effect of the traditional least squares method and ensure the convergence performance. The target equation was given as follows:

$$
\phi(W)=(\widehat{G}-W)^{\prime} \Sigma^{-1}(\widehat{G}-W)+\beta K(w) .
$$

In (4), $W$ represented the ideal value of the estimated projection data, $\Sigma^{-1}$ represented the noise variance matrix, $\widehat{G}$ was the projection data measured by the reagent, and the last term $K(w)$ was the marked penalty factor, which can control the convergence performance, and $\beta$ could adjust the degree of calculation.

If it was supposed that $i$ represented the guide value of each pixel on the image, $J_{i}$ was the pixel value at the center of the image, $Y_{j}$ represented the value of adjacent pixels, and $A_{i j}$ referred to the weight, then the final iterative equation was as follows:

$$
W_{i}^{(n+1)}=\frac{W_{i}+\beta \partial_{i}^{2}\left(\sum_{k \in J_{i}^{2}} A_{i j} Y_{j}^{n+1}+\sum_{k \in J_{i}^{2}} A_{i j} Y_{j}^{n}\right)}{1+\beta \partial_{i}^{2} \sum_{k \in J_{i}^{2}} A_{i j}} .
$$

In the previous equation, $k$ represented the iterative period of the equation and $A$ and $Y$ were the adjacent areas of the front, back, left, and right centered on the target pixel. The relationship between the preprocessed projection data and the estimated projection data was as follows:

$$
\begin{aligned}
T(W) & =\frac{1}{2} \sum \sum_{\substack{\mathrm{NuJ}_{i} \\
i}} A_{i j}\left(W_{i}-Y_{j}\right), \\
W & =\arg \min _{p \geq 0} \varphi(W) .
\end{aligned}
$$

2.5. Evaluation Indicators. In this study, the HIR algorithm was compared with the filtered backprojection reconstruction (FBPR) algorithm by measuring the image noise of the reconstructed image of the algorithm and selecting the accuracy and sensitivity as indicators. The specific equation was given as follows:

$$
\begin{aligned}
& A=\frac{F_{T}}{T} \times 100 \%, \\
& S=\frac{P}{W+F} \times 100 \%, \\
& Y=\frac{N}{W+F} \times 100 \% .
\end{aligned}
$$

In (8) (10), $A, S$, and $Y$ referred to the accuracy, sensitivity, and specificity, respectively, $F_{T}$ represented the number of accurate predictions, $T$ represented the total number of patients, $P$ referred to the number of patient with true positive, $N$ and $F$ referred to the number of patients with 
true negative and false positive, respectively, and $W$ meant the number of patients with false negative.

2.6. Image Reconstruction and Effect Evaluation. In this research, the optimized iterative reconstruction algorithm was used to reconstruct CT images of cerebral aneurysms, and it was used to solve the problem of image sharpness. In addition, backprojection image reconstruction algorithm and Fourier transform analytic method were introduced. According to the CT images of cerebral arteries of patients, the lesions were presented in a three-dimensional and visual way through the reconstructed three-dimensional images, thus achieving the effects of simulation and simulation.

2.7. Statistical Methods. The data processing of this study was analyzed by SPSS19.0 version statistical software. Measurement data were expressed as mean \pm standard deviation $(\bar{x} \pm s)$, count data were expressed in the form of percentage $(\%)$, and the $t$-test and $\chi^{2}$ test were used for comparison, respectively. Variance test was used for the treatment success rate, second surgery rate, and complications of the routine group and intervention group. $P<0.05$ meant that the difference was statistically significant.

\section{Results}

3.1. Classification Performance of HIR Algorithm. Figure 4 shows the comparison results of the sensitivity, specificity, accuracy, and running time of the optimized iterative reconstruction algorithm with the backprojection image reconstruction algorithm and Fourier transform analytical method. It can be concluded that the sensitivity, specificity, and accuracy of the hybrid iterative reconstruction algorithm were $90.78 \%, 83.27 \%$, and $94.82 \%$, and the running time was $15.18 \pm 2.19 \mathrm{~s}$. The sensitivity, specificity, and accuracy of the hybrid iterative reconstruction method were higher than those of the backprojection image reconstruction algorithm and Fourier transform analysis method, but the running time was shorter $(P<0.05)$, which showed that CT images under this algorithm could improve doctors' diagnosis.

3.2. Basic Data of Patients. A total of 50 patients with cerebral aneurysm were selected as the research objects, including 27 males and 23 females, with an average age of $57.3 \pm 4.72$ years old. One of them was a 55 -year-old male patient with an intracranial aneurysm of the internal carotid artery. The general information of patients is shown in Figure 5.

Figures 6 and 7 show the schematic diagrams of cerebral aneurysm formation and surgery, and Figure 8 shows the CT image and 3D reconstruction image of sample patient (55-year-old male patient) with cerebral aneurysm. It can be concluded that the $3 \mathrm{D}$ reconstruction imaging of the cerebral aneurysm of the patient's middle cerebral artery showed that the aneurysm was located at the early frontal cortical branch (EFCB).
3.3. Simulation Analysis of Cerebral Aneurysm before and after Stent Implantation. Figures 9 and 10 shows that the blood flow velocity in the neck and the outlet before stent implantation were $7.35 \times 10^{-2} \mathrm{~m} / \mathrm{s}$ and $1.51 \times 10^{-1} \mathrm{~m} / \mathrm{s}$, respectively, the maximum velocity appeared in the upstream part of the outlet, and the blood flow velocity began to decrease gradually after passing through the aneurysm, forming a whirlpool at the top of the tumor. After implantation of the stent, the flow velocities of cervical aneurysm at the neck and exit were $9.352 \times 10^{-2} \mathrm{~m} / \mathrm{s}$ and $1.897 \times 10^{-2} \mathrm{~m} / \mathrm{s}$, respectively. Although the blood flow velocity changed greatly, the blood flow velocity in the aneurysm did not increase dramatically. But the maximum velocity appeared in the area below the neck of the tumor, the blood flow slowed down after entering the aneurysm, and there was no vortex at the top of the tumor. The wall shear stress distribution revealed that the shear stress on the cervical aneurysm neck wall before stent implantation was larger, and the maximum wall shear stress reached $3.187 .35 \times 10^{3} \mathrm{~Pa}$. Compared with the wall shear stress at the blood flow impact site, the shear stress at the exit, the top of the tumor, and the surface of the tumor were relatively small. After stent implantation, the wall shear stress area on the far side of the tumor neck was significantly reduced, the blood flow at the top of the tumor body flowed slowly, and the wall shear stress gradually decreased and disappeared. Theoretically, the greater the shear stress, the stronger the material's ability to resist damage. Therefore, the greater the shear stress of the tumor neck before implantation, the less the thickening of the tumor neck and the wall of the tumorbearing artery, which would eventually cause the top of the tumor to rupture. However, the shear stress in the neck of the aneurysm was weakened after implantation, which reduced the possibility of cerebral aneurysm rupture. The wall pressure distribution disclosed that before implantation, the entrance wall pressure was larger and the pressure on the tumor wall also increases until the pressure at the back of the tumor begins to gradually decrease due to the influence of blood flow on the artery of the tumor structure. After the stent implantation, the pressure on the tumor wall did not change greatly; that is to say, the stent implantation exerted little effect on the pressure value of the distal wall and top of the tumor neck at any time.

In the absence of residual stress, the wall stresses at the entrance and exit before stent implantation and after stent implantation were basically the same, but the changes in cervical aneurysm neck and top were relatively great after implantation of the stent. The reason for the change may be the fact that cerebral aneurysm stent implantation can better reduce the rupture of tumor wall due to the impact of blood flow. When the residual stress was considered, the deformation of the aneurysm wall was the same as the case without considering the residual stress. The maximum deformation before implantation without considering residual stress was $8.152 \times 10^{-2} \mathrm{~mm}$, and the maximum deformation after implantation was $1.073 \times 10^{-2} \mathrm{~mm}$, while the maximum deformations before and after the stent implantation considering residual stress were $1.389 \times 10^{-1} \mathrm{~mm}$ and $1.998 \times 10^{-1} \mathrm{~mm}$, respectively. Based on the above, the wall 


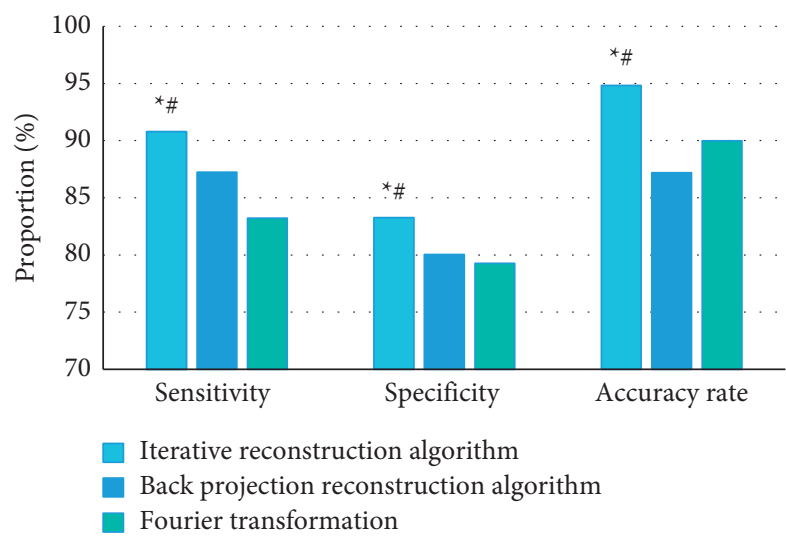

(a)

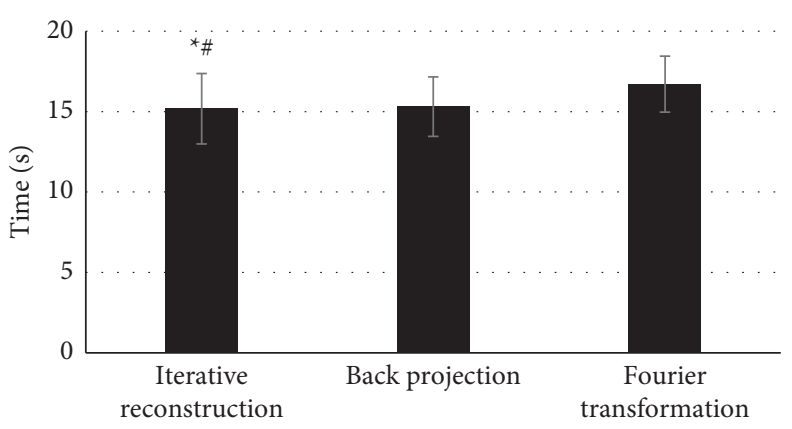

(b)

Figure 4: (a) Comparison of sensitivity and specificity of hybrid iterative algorithm; (b) algorithm running time. ${ }^{*}$ means the difference is statistically significant compared with the backprojection reconstruction algorithm $(P<0.05)$; \# indicates the difference is statistically significant compared with Fourier transform analysis $(P<0.05)$.

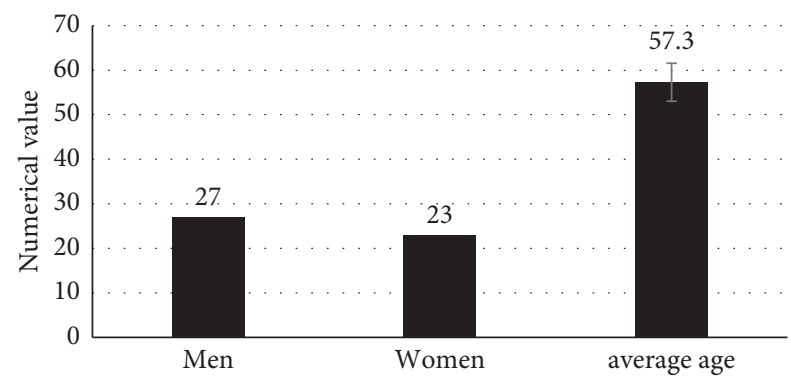

FIGURE 5: Age, gender ratio, and hospital stays of the patients in two groups.

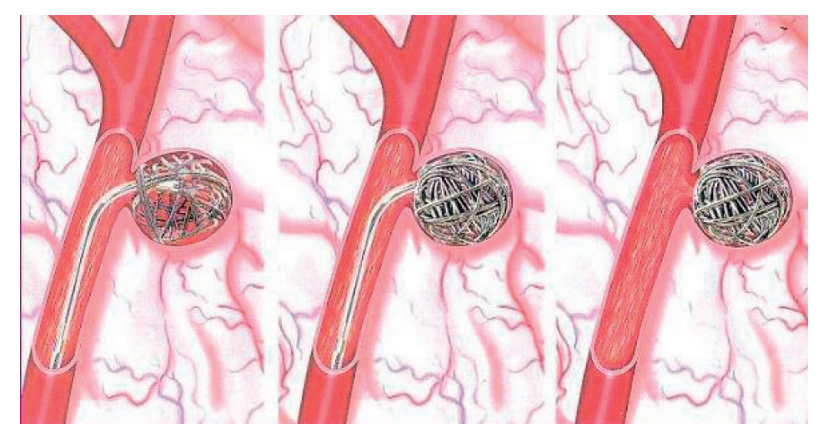

FIgURE 6: Forming of cerebral aneurysm.

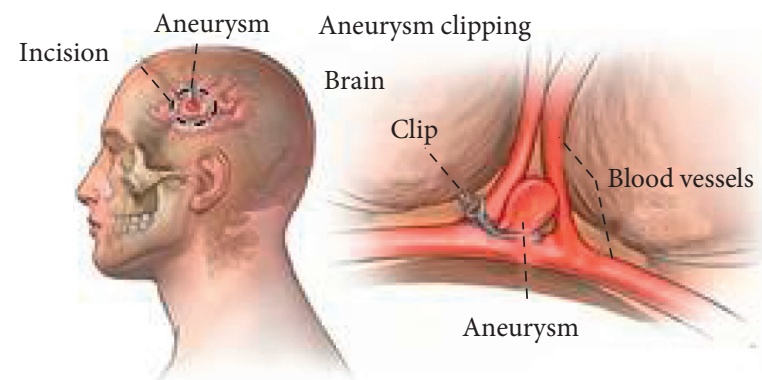

FIGURE 7: Schematic diagram of cerebral aneurysm and surgery. 


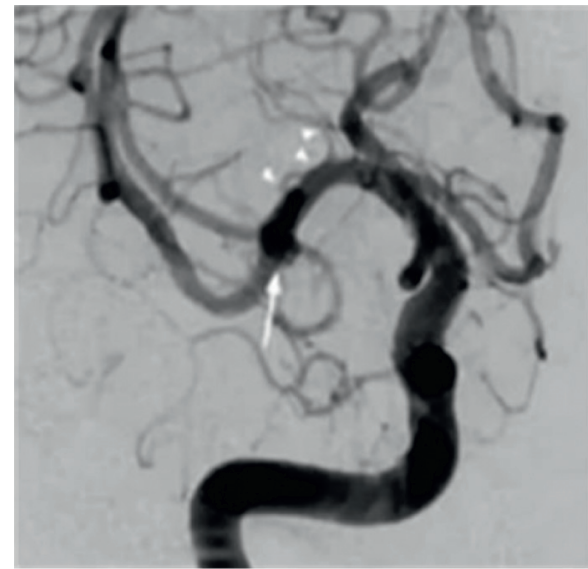

(a)

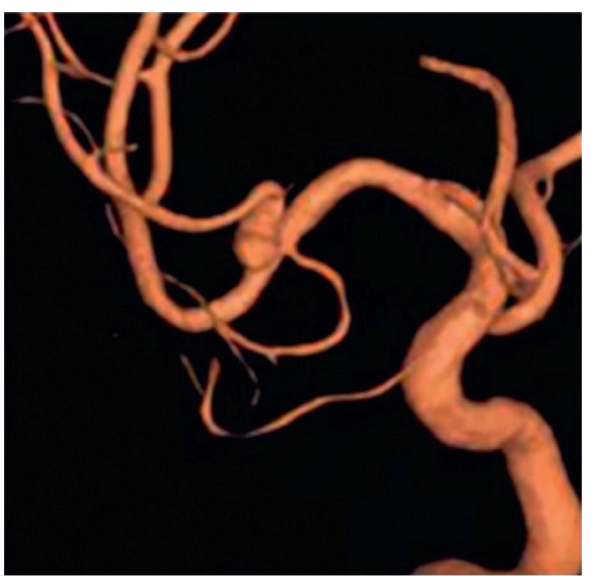

(b)

FIGURE 8: The middle cerebral artery (MCA) cerebral aneurysm of a 55-year-old male patient (a); preoperative DSA and its 3D reconstruction imaging showed that the aneurysm was located at EFCB (b).
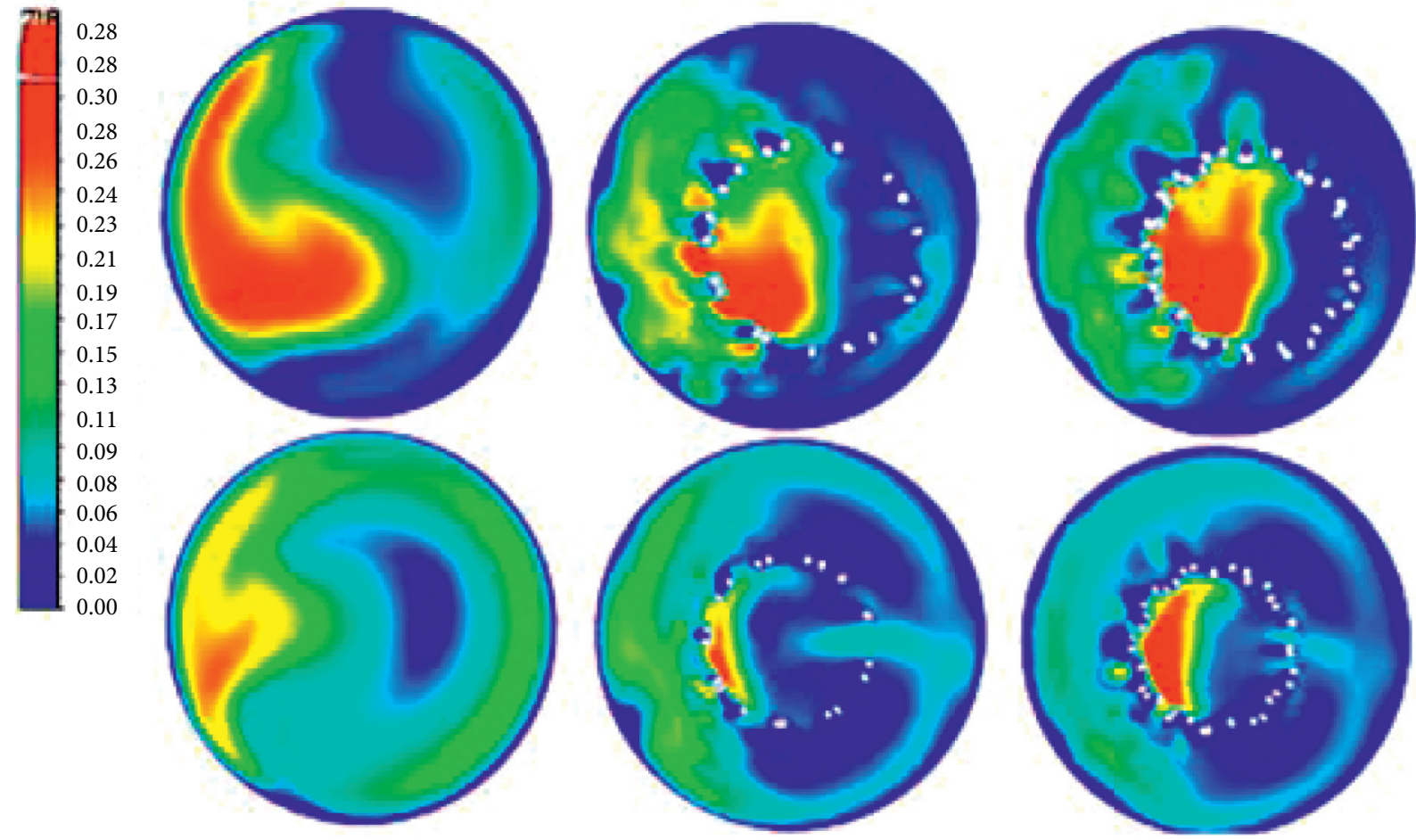

Figure 9: Pressure distribution of cerebral aneurysm.

deformation of cerebral aneurysm in consideration of the residual stress was greater than that without considering the residual stress under the same circumstances. Therefore, calculating the tumor wall deformation based on the clinical consideration of the vascular residual stress was more in line with the actual situation.

3.4. Clinical Analysis afterStent Implantation. In this study, a 55-year-old male patient with an intracranial aneurysm of the internal carotid artery showed preoperative DSA angiography and showed vascular stenosis at the beginning of the aneurysm. After the spiral stent implantation vessel designed in this study was applied, the CT imaging was performed again. The results showed that the arterial vessel had better dilatation, the diameter of the vessel was significantly enlarged, and the blood flow velocity was improved to a certain extent (Figure 11). From a clinical point of view, the symptoms of dizziness, numbness, and weakness had also been relieved to a certain extent. The clinical treatment effect verified the feasibility of the simulation analysis method and the reliability of the aneurysm flow field dynamics data.

Figure 12 shows the numerical analysis and comparison of 8 cases of cerebral artery patients before and after stent 

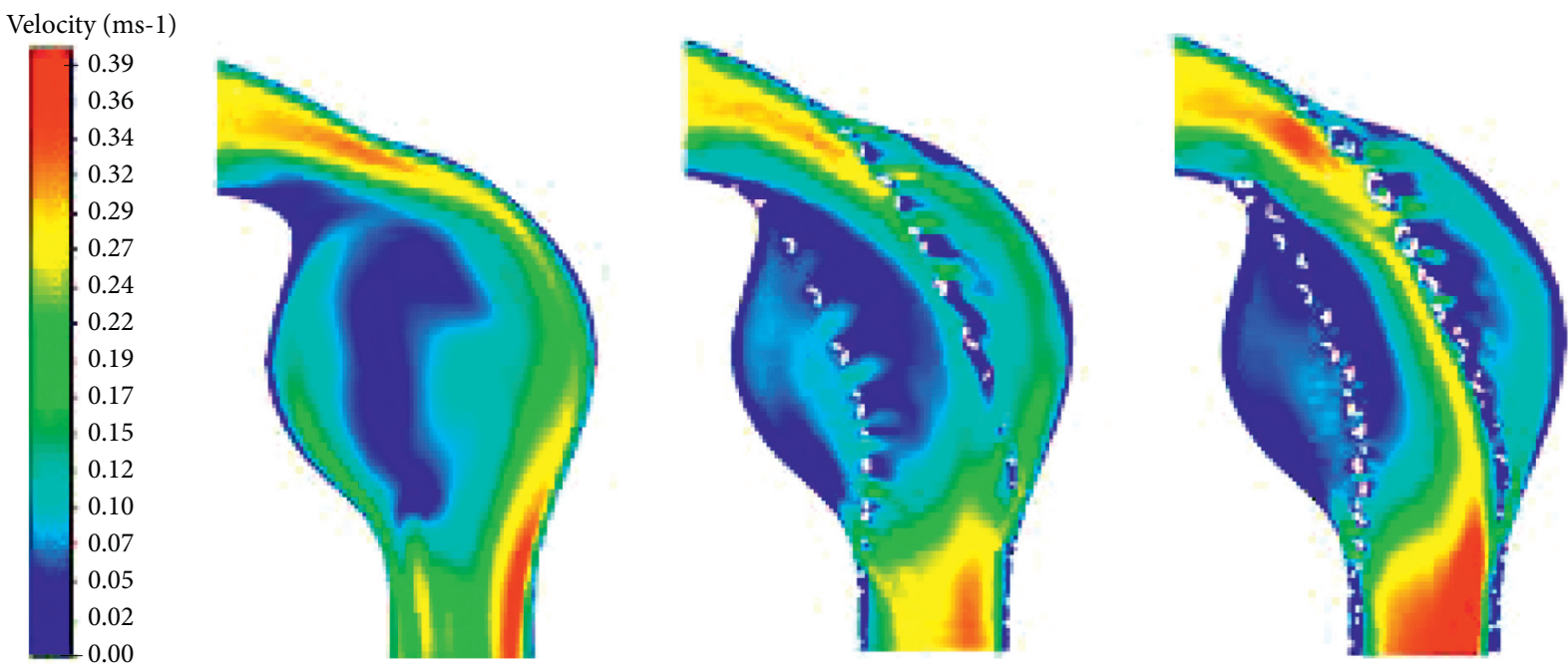

FIgURE 10: Flow velocity distribution of cerebral aneurysm.

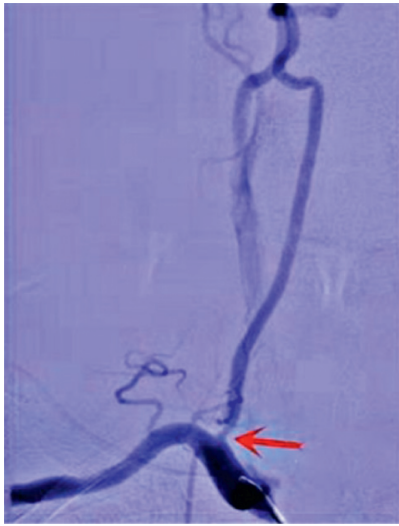

(a)

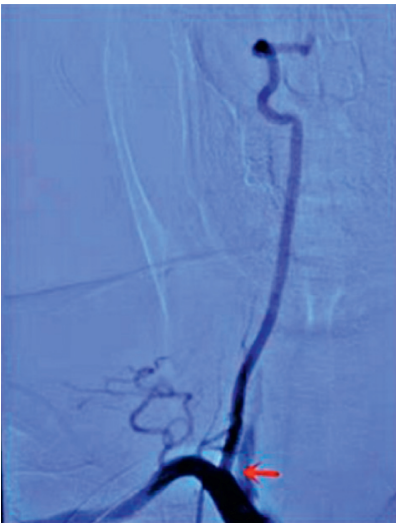

(b)

Figure 11: Comparison of cerebral arteries and blood vessels before and after the stent implantation: (a) the image before stent implantation; (b) the image after stent implantation.

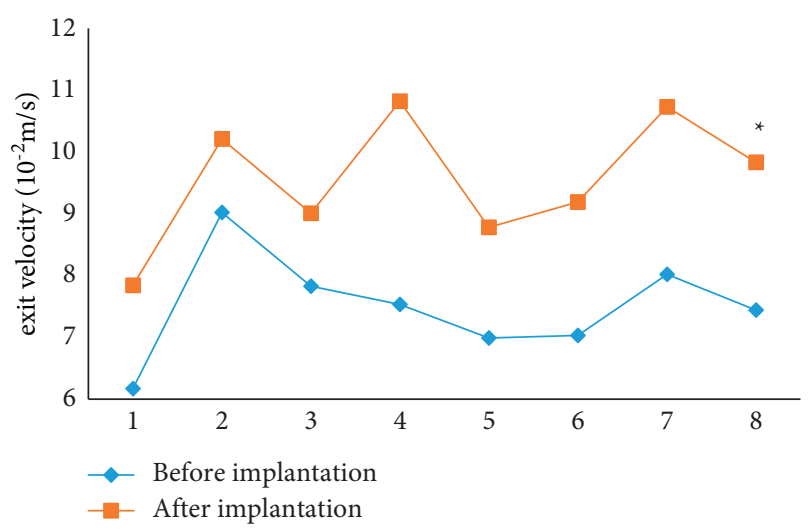

Figure 12: Comparison of the flow velocities of the arterial vessel outlet in 8 patients. Note: ${ }^{*}$ suggested that the difference was statistically obvious in contrast to the value before stent implantation. implantation. The figure suggested that the flow rate before stent implantation was much lower than that after stent implantation, and the difference was statistically obvious $(P<0.05)$. The wall shear stress distribution of the cerebral aneurysm tumor of the patient before implantation of the stent showed an obvious decreasing trend compared with that before implantation, showing statistical difference $(P<0.05)$ (Figure 13). In addition, the pressure distribution of the wall did not change greatly. After stent implantation, the degree of deformation of the cerebral aneurysm wall surface had been improved, making the aneurysm not easy to rupture. The clinical treatment results were similar to those of the simulation experiment, which indicated that the poor blood flow of cerebral aneurysm can be dramatically improved after the stent was implanted. The influence of the morphology of the tumor wall on the tumor wall was to analyze the rupture mechanism of cerebral aneurysm, which can provide theoretical support and reference for clinical diagnosis and treatment. 


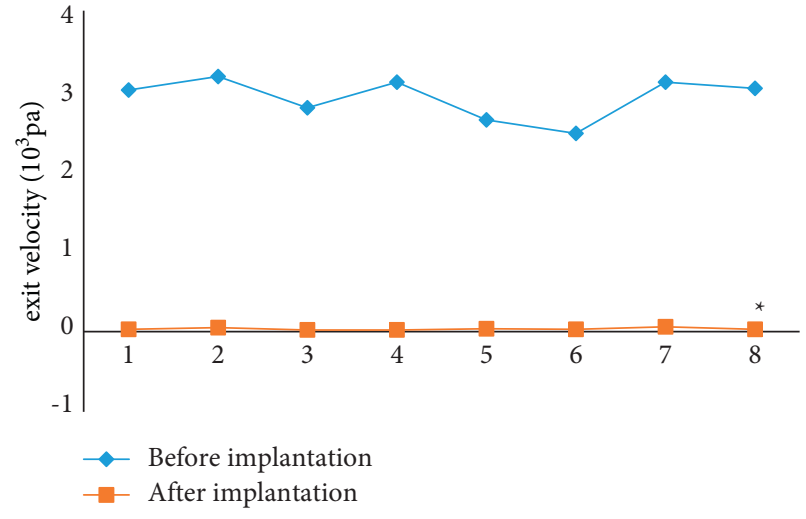

FIGURE 13: Comparison on maximal shear stress of cerebral aneurysm in 8 patients. Note: ${ }^{*}$ suggested that the difference was statistically obvious in contrast to the value before stent implantation.

\section{Discussion}

Intracerebral stent implantation is the most commonly used technique for the treatment of cerebral aneurysms, but whether the hemodynamic parameters of blood flow field are improved and whether the possibility of aneurysm rupture after stent implantation is reduced are still the most important clinical problem at present. Based on the optimized hybrid iterative algorithm, this research carried out 3D modeling of cerebral aneurysm from CT image data, conducted transient bidirectional fixed coupling blood flow simulation, and obtained the corresponding hemodynamic parameters after stent implantation. The results showed that the sensitivity, specificity, and accuracy of the hybrid iterative algorithm were $90.78 \%, 83.27 \%$, and $94.82 \%$ and the running time was $15.18 \pm 2.19 \mathrm{~s}$. The sensitivity, specificity, and accuracy of the hybrid iterative reconstruction method were higher than those of the backprojection image reconstruction algorithm and Fourier transform analysis method, but the running time was shorter $(P<0.05)$, which was similar to Dunet et al. [14].

Karttunen et al. [15] used CT data combined with Mimics image processing software to three-dimensionally reconstruct the solid aneurysm structure and applied the finite element analysis software to obtain an aneurysm hemodynamic distribution map. Otani et al. [16] did a steady flow analysis on the cerebral aneurysm discontinuous growth model to obtain the utility and regular changes of hemodynamic factors in the growth process of the tumor, providing a reference for the analysis of aneurysm growth and rupture. Mori et al. [17] studied the numerical simulation methods to compare cerebral aneurysm internal hemodynamic parameters and cerebral aneurysm biological behaviors under different conditions, showing high clinical significance. In this study, blood flow velocity in the neck and the outlet before stent implantation were $7.35 \times 10^{-2} \mathrm{~m} / \mathrm{s}$ and $1.51 \times 10^{-1} \mathrm{~m} / \mathrm{s}$, respectively, the maximum velocity appeared in the upstream part of the outlet, and the blood flow velocity began to decrease gradually after passing through the aneurysm, forming a whirlpool at the top of the tumor. After implantation of the stent, the flow velocities of cervical aneurysm at the neck and exit were $9.352 \times 10^{-2} \mathrm{~m} / \mathrm{s}$ and $1.897 \times 10^{-2} \mathrm{~m} / \mathrm{s}$, respectively. The blood flow slowed down after entering the aneurysm, and there was no vortex at the top of the aneurysm. Before stent implantation, the shear stress on the cervical aneurysm neck wall was larger, and the maximum shear stress on the wall reaches $3.187 .35 \times 10^{3} \mathrm{~Pa}$. Compared with the wall shear stress at the blood flow impact site, the shear stress at the exit, the top of the tumor, and the surface of the tumor were relatively small. After stent implantation, the wall shear stress area on the far side of the tumor neck was significantly reduced, the blood flow at the top of the tumor body flowed slowly, and the wall shear stress gradually decreased and disappeared. The maximum deformation before implantation without considering residual stress was $8.152 \times 10^{-2} \mathrm{~mm}$, and the maximum deformation after implantation was $1.073 \times 10^{-2} \mathrm{~mm}$, while the maximum deformations before and after the stent implantation considering residual stress were $1.389 \times 10^{-1} \mathrm{~mm}$ and $1.998 \times 10^{-1} \mathrm{~mm}$, respectively. Based on the above, the wall deformation of cerebral aneurysm in consideration of the residual stress was greater than that without considering the residual stress under the same circumstances. Therefore, calculating the tumor wall deformation based on the clinical consideration of the vascular residual stress was more in line with the actual situation. The flow rate before stent implantation was much lower than that after stent implantation, and the difference was statistically obvious $(P<0.05)$. The wall shear stress distribution of the cerebral aneurysm tumor of the patient before implantation of the stent showed an obvious decreasing trend compared with that before implantation, showing statistical difference $(P<0.05)$. The results of this study were highly similar to the conclusions of Feng and Shu [18], indicating that after the stent implantation, the hemodynamic parameters of the cerebral arteries had changed greatly compared with those before the stent implantation. The implantation of the stent can obviously reduce the wall shear force of the aneurysm neck, which can improve the aneurysm blood flow field distribution.

\section{Conclusion}

An optimized HIR algorithm was constructed and applied to the $3 \mathrm{D}$ reconstruction of $\mathrm{CT}$ image data after its sensitivity, accuracy, and specificity were tested. It was found that CT image analysis based on the HIR algorithm revealed that stent implantation can greatly reduce the shear force of the aneurysm neck and improve the distribution of the aneurysm's blood flow field. Wall shear force was one of the main hemodynamic factors leading to the rupture of the cerebral aneurysm. This study could provide reference for further research on complex cerebral aneurysm and its treatment plan. However, there were some shortcomings for this study. The experiment process lacked the control experiment of other algorithms. Therefore, the experimental results had a certain degree of subjectivity. In addition, there were fewer research samples, and the test results were not suitable for large-scale use. In the future work, it will further conduct 
comparative experiments on it to fully understand the optimization method of CT images under this algorithm, so as to obtain more perfect results and theoretical basis. In summary, pelvic ultrasound based on the HIR algorithm showed a high diagnostic accuracy for changes in blood flow field in cerebral aneurysm, and applying of CT images to the diagnosis of cerebral aneurysm can objectively provide clinical imaging data, showing high application value.

\section{Data Availability}

The data used to support the findings of this study are available from the corresponding author upon request.

\section{Conflicts of Interest}

The authors declare no conflicts of interest.

\section{Authors' Contributions}

Kunyang Bao and Chao Liu contributed equally to this work.

\section{Acknowledgments}

This work was supported by Scientific Research Project of the Health Commission of Sichuan Province (no. 19PJ296).

\section{References}

[1] H. Kuroda, T. Mochizuki, S. Shimizu, and T. Kumabe, "Rupture of thrombosed cerebral aneurysm during antithrombotic therapy for ischemic stroke: case report and literature review," World Neurosurgery, vol. 126, pp. 468-471, 2019.

[2] Z. Lv and W. Xiu, "Interaction of edge-cloud computing based on SDN and NFV for next generation IoT," IEEE Internet of Things Journal, vol. 7, no. 7, pp. 5706-5712, 2020.

[3] M. Desai, A. R. Wali, H. S. Birk, D. R. Santiago-Dieppa, and A. A. Khalessi, "Role of pregnancy and female sex steroids on aneurysm formation, growth, and rupture: a systematic review of the literature," Neurosurgical Focus, vol. 47, no. 1, p. E8, 2019.

[4] J. D. Sokolowski, E. L. Guilliams, M. Diaz et al., "Neoplastic cerebral aneurysm from metastatic lung adenocarcinoma with neuroendocrine features," World Neurosurgery, vol. 122, pp. 155-160, 2019.

[5] S. Takubo, K. Kawasaki, T. Nagatari, M. Matsumoto, and T. Kageyama, "Clinical usefulness of ultra-short TE MRA for follow-up imaging after cerebral aneurysm clipping," Japanese Journal of Radiological Technology, vol. 76, no. 2, pp. 177-184, 2020.

[6] X. Gao and J. Cai, "Optimization analysis of urban function regional planning based on big data and GIS Technology," Boletin Tecnico/Technical Bulletin, vol. 55, no. 11, pp. 344-351, 2017.

[7] W. Venderink, F. van Megen, J. de Vries, and F. J. A. Meijer, "Cerebral aneurysm rebleed with ventricular breakthrough captured by four-dimensional CT angiography," Neuroradiology, vol. 60, no. 7, pp. 665-667, 2018.

[8] M. Katsura, J. Sato, M. Akahane et al., "Single-energy metal artifact reduction technique for reducing metallic coil artifacts on post-interventional cerebral CT and CT angiography," Neuroradiology, vol. 60, no. 11, pp. 1141-1150, 2018.
[9] S. D. Hajdu, R. T. Daniel, R. A. Meuli, J.-B. Zerlauth, and V. Dunet, "Impact of model-based iterative reconstruction (MBIR) on image quality in cerebral CT angiography before and after intracranial aneurysm treatment," European Journal of Radiology, vol. 102, pp. 109-114, 2018.

[10] T. Struffert, S. Lang, E. Adamek, T. Engelhorn, C. M. Strother, and A. Doerfler, "Angiographic C-arm CT visualization of the Woven EndoBridge cerebral aneurysm embolization device (WEB): first experience in an animal aneurysm model," Clinical Neuroradiology, vol. 24, no. 1, pp. 43-49, 2014.

[11] N. Shimooka, S. Ishida, and M. Kawai, "[Ruptured aneurysm of bihemispheric anterior cerebral artery A1-A2 junction:A case report]," Noshinkeigeka, vol. 45, no. 3, pp. 253-257, 2017.

[12] O. S. Grosser, J. Ruf, D. Kupitz et al., "Iterative CT reconstruction in abdominal low-dose CT used for hybrid SPECT-CT applications: effect on image quality, image noise, detectability, and reader's confidence," Acta Radiologica Open, vol. 8, no. 6, p. 205846011985626, Article ID 2058460119856266, 2019.

[13] S. X. Xie, Z. C. Yu, and Z. H. Lv, "Multi-disease prediction based on deep learning: a survey," Computer Modeling in Engineering and Sciences, vol. 127, no. 3, pp. 1-34, 2021.

[14] V. Dunet, M. Bernasconi, S. D. Hajdu, R. A. Meuli, R. T. Daniel, and J.-B. Zerlauth, "Impact of metal artifact reduction software on image quality of gemstone spectral imaging dual-energy cerebral CT angiography after intracranial aneurysm clipping," Neuroradiology, vol. 59, no. 9, pp. 845-852, 2017.

[15] A. I. Karttunen, P. H. Jartti, V. A. Ukkola, J. Sajanti, and M. Haapea, "Value of the quantity and distribution of subarachnoid haemorrhage on CT in the localization of a ruptured cerebral aneurysm," Acta Neurochirurgica, vol. 145, no. 8, pp. 655-661, 2003.

[16] T. Otani, A. Al-Issa, A. Pourmorteza, E. R. McVeigh, S. Wada, and H. Ashikaga, "A computational framework for personalized blood flow analysis in the human left atrium," Annals of Biomedical Engineering, vol. 44, no. 11, pp. 3284-3294, 2016.

[17] K. Mori, T. Saida, F. Sato et al., "Endoleak detection after endovascular aneurysm repair using unenhanced MRI with flow suppression technique: feasibility study in comparison with contrast-enhanced CT," European Radiology, vol. 27, no. 1, pp. 336-344, 2017 Jan.

[18] Y. Feng and S. J. Shu, "Diagnostic value of low-dose 256-slice spiral CT angiography, MR angiography, and 3D-DSA in cerebral aneurysms," Disease Markers, vol. 2020, pp. 1-5, 2020. 